\title{
Implementation of Industry 4.0 in the belt conveyor transport
}

\author{
Gabriel Fedorko ${ }^{1, *}$ \\ ${ }^{1}$ TU Košice F BERG, Logistics Department, Park Komenského 14, 04001 Košice, Slovakia
}

\begin{abstract}
Industry 4.0 is gradually penetrating into different industries that are starting to apply this concept intensively. This brings a wide range of options that need to be exploited effectively. The list of areas in which Industry 4.0 can find its use is expanding. At present, it can be said that there is practically no area in which this concept could not be applied. For its successful implementation, it is therefore necessary to look for application options and to analyze and explore individual areas. Belt conveyor transport is one of the domains that need to get ready for the implementation of the Industry 4. The implementation of the concept in this industry applies predominantly on the deployment of CPS' Internet of things and Internet of service in the operation of the belt conveyor and, consequently, the entire transport system. This paper presents the issue with a focus on the field of belt conveyor transport.
\end{abstract}

\section{Introduction}

Belt conveyor transport is currently one of the most important systems of intra-company transport in a wide range of industries. In order to facilitate its reliable operation it is necessary to continuously carry out research that is targeted at various types of problem areas. Presently, priority areas of research include issues related to automation of individual processes, predictive maintenance and efficient operation.

One of the basic conditions for its reliable implementation is the monitoring of the entire transport process. In addition to the classic approach, this requirement can also be met with a more convenient form of online monitoring.

This approach also allows monitoring the transport on the conveyor belt [1]. With its help, it is possible to monitor continuously the size of the material particles transported to obtain a huge amount of data. This method can also be considered as highly efficient in terms of further development of the belt conveyor transport technology used for the transport of different types of materials.

The data obtained together with other parameters can be used primarily for the maintenance of belt conveyors [2]. Further use of the data obtained is possible, for example, in the implementation of various decision-making processes [3]. The monitoring of the operation of belt conveyor transport thus progresses to the forefront as a trend indicating the direction of further development of the belt conveyor transport sector [4]. The ideas of a

\footnotetext{
* Corresponding author: gabriel.fedorko@tuke.sk
} 
smart conveyor belt [5] are also emerging. [5]. It can therefore be argued that the belt conveyor transport sector is gradually getting closer to alignment with the concept of Industry 4.0 .

\section{Description of Industry 4.0}

Industry 4.0 is an issue that is currently receiving a growing attention in a wide range of industries. Since its introduction in 2011 by the German research and development association for science and economics it has undergone progressive and increasingly intensive developments. Its first application was planned as a part of a research program aimed at maintaining Germany at a high technological level. At present, however, Industry 4.0 has already crossed the German border and is becoming the dominant industry concept, which is expected to grow significantly after 2020.

After initial and often debatable applications, the possibilities of its application, especially in the automotive and electrical engineering industry, gradually developed. These are areas characterized by a high degree of mass production. At present, however, the industry 4.0 concept and its partial tools are slowly being promoted in other industrial sectors, in the form of cyber - physical systems.

\subsection{Cyber - physical system}

Cyber - physical system (CPS) is the operational basis of Industry 4.0. It combines two key components of this concept: the internet of Things (IoT) and the Internet of Services (IoS). Specifically, CPS combined with IoT and IoS creates a base for Industry 4.0.

This combination can be understood as a network in which different information is continuously processed by various powerful software tools and specialized user interfaces. CPS can be expressed in graphical form as a triangle whose vertices represent three components - computation, communication and control (Fig. 1).

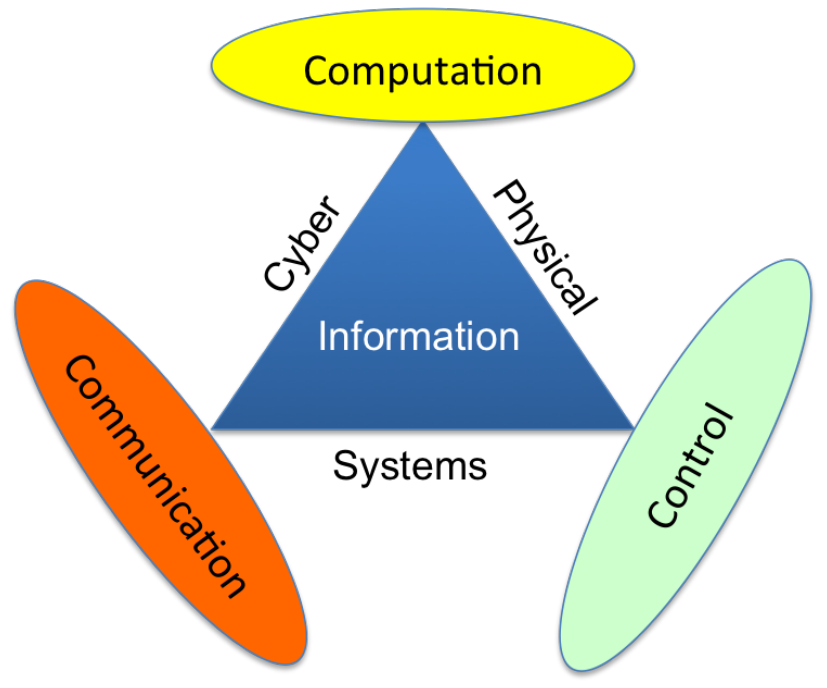

Fig. 1. CPS Principle. Source: [6]

According to [7], CPSs are broadly categorized according to the following categorization: 
- $1^{\text {st }}$ generation CPSs contain identification technologies such as RF Frequency Identification (RFID) sensors that enable a unique identification of the device in which they are fitted,

- $2^{\text {nd }}$ generation CPSs are equipped with sensors and actuators with a limited range of functions

- $3^{\text {rd }}$ Generation CPSs can store, analyze, and process data over a relatively large scale, and are equipped with multiple sensors and actuators.

\subsection{Belt conveyor as Cyber - physical system}

The CPS presents the integration of communication and computational structures and tools with individual physical processes. The main difference between the CPS and the embedded systems is the fact that CPSs are not separate devices, but networks of interconnected devices that communicate with each other at a certain level.

A common embedded system is mostly a monitoring (control) unit that works with several sensors. However, in order to meet the requirements for CPS, it is necessary for the nested systems to define a communication interface that will provide data exchange and data communication. Data can be intended for further use in other devices or storage in the form of cloud storage. This means that data exchange over the network is done by embedded CPS [8].

It follows from the above that if we want any conveyor belt to be considered a CPS, then we need to have standard monitoring tools and devices connected to the network and to conduct bi-directional data communication. This requires various existing embedded systems (Fig. 2) or the design of new such systems.
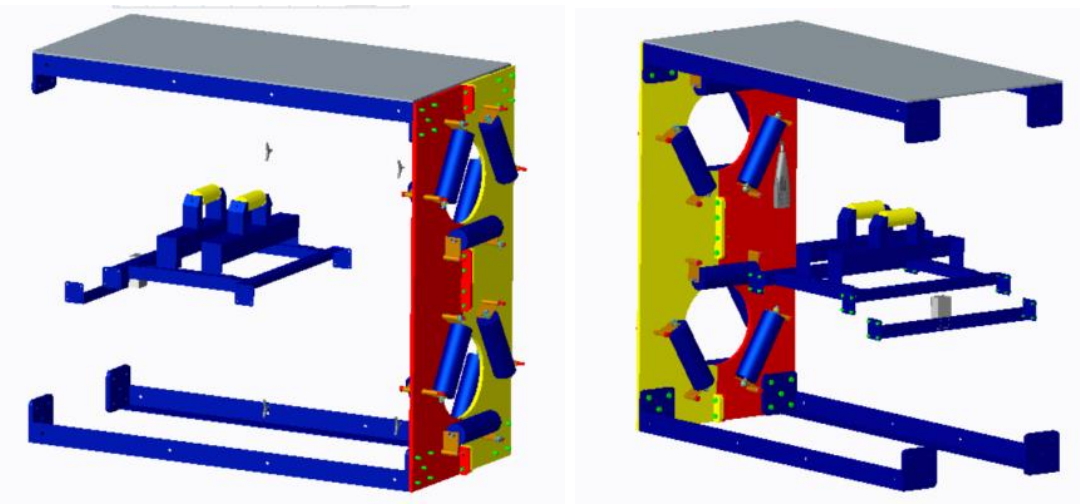

Fig. 2. Example of embedded system for belt conveyor transport - smart roller idler. Source: Autor

\subsection{Smart conveyor}

Smart conveyor is a CPS consisting of any type of conveyor that performs two-way data communication using a suitable communication protocol. Within this communication, the data is transmitted in a compatible form and depending on the operation of the smart conveyor. These data are then processed and evaluated in a suitable system. This process is aimed at obtaining a wide range of different indicators providing information on the belt conveyor operation process. From the obtained and assessed data, the control sequences can be generated by means of an expert control system. These are subsequently sent back over the appropriate communication interface to the conveyor control unit (Fig. 3). 


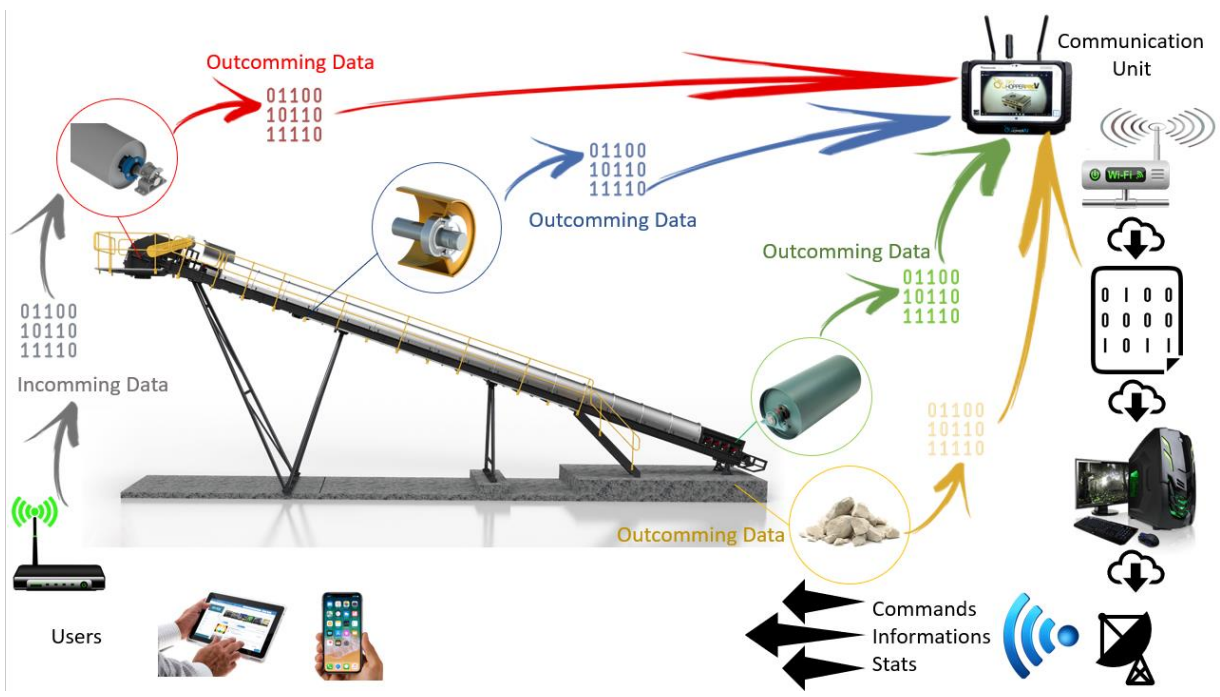

Fig. 3. Smart conveyor. Source: Autor

Such a CPS can be classified as a holonic CPS. The Smart Conveyor is in principle a multiagent system because it is made up of individual structural elements that are fitted with sensing and control elements. Such a holonic agent is able to plan, coordinate, or even actively perform an activity within an intelligent conveyor.

\section{Application of holonic agents within an intelligent conveyor}

Several options for creating holonic agents within an intelligent conveyor can be considered, e.g. rollers in a roller idler, or a drum drive unit can be mentioned.

\subsection{Roller}

The roller as one of the basic parts of the belt conveyor is of a simple design (Fig. 4).

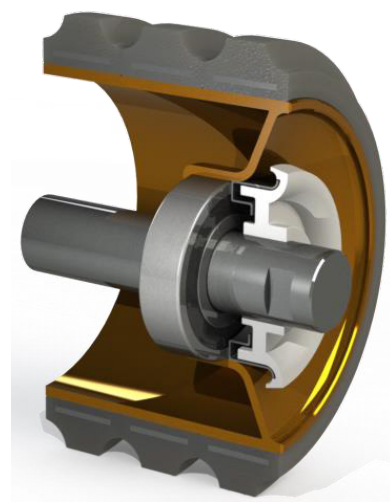

Fig. 4. A cross-sectional view of a belt conveyor roller. Source: [9]

Within the CPS, the roller is capable of providing a variety of information, e.g. rotation speed, bearing temperature, contact pressure, etc. Using this information, it is possible to monitor, for example, its durability [10-11]. This means that the roller, thanks to its sensor, will transmit a multitude of data about the course of its operation, which will be assessed 
and compared with the defined measurement standard by the respective control unit. If the received data deviate from the measurement standard indicating trouble-free operation, the control unit sends a signal to the roller and it will signal the fault condition and the need for operator intervention.

This means that the fault condition is self-diagnosed and the intervention of the operator will be performed on the basis of precise instructions and identification of the fault site. This procedure can be used in various situations including roller duration monitoring, maintenance planning, monitoring of tribological aspects, etc.

However, for the implementation of such belt conveyor operation, it is still necessary to solve a whole range of difficulties in the future in order to make the whole process possible.

\subsection{Drum drive unit}

The drive unit is another type of holonic agent within a smart conveyor. In addition to similar functionality to the roller, it is possible to enable this agent to stop the entire smart conveyor if necessary. The Stop can be done either on the basis of mutual data communication between the individual holonic agents, or on the basis of an order sent from the control unit. Within this type of holonic agent, a certain subset of CPSs can also be applied in the form of an embedded system that will be equipped with a control algorithm and a calculating unit, but this system must also be actively connected to the communication network of the entire system.

\section{Conclusion}

Industry 4.0 is no longer just a concept that addresses a narrow spectrum of industrial areas. On the contrary, it is increasingly necessary to look for further possibilities for its application. Such areas include the transport of materials by various types of conveyors. This process can not in any way be limited by the fact that at some point it is still not possible to carry out some of the requirements because it is a secondary problem. The primary task is to look for ways to implement and exploit Industry 4.0 ideas for conveyor issues. The main goal to keep in mind is to make work easier and more efficient. In this way, the conveyor can be a fully autonomous device that will be able to communicate with other devices and systems. The result of such communication will then penetrate into various areas of enterprise logistics and make it more efficient.

The present paper is a part of research grant projects VEGA 1/0063/16, VEGA 1/0403/18, KEGA 018TUKE-4/2016.

\section{References}

1. M. Soprana, A.C. Santomaso, P. Facco, 28 th. E. Symp. C. A. Proc. 43, 6 (2018)

2. P. Stefaniak, J. Wodecki, R. Zimroz, Adv. Tech. Diag. 10, 12 (2018)

3. S. Jovcic, P. Prusa, S. Nikolicic, Adv. Sci. Tech. J. 12, 7 (2018)

4. X. Liu, Y. Pang, G. Lodewijks, D. He, Measur. 127, 6 (2018)

5. L. Jurdziak, R. Blazej, M. Bajda, Adv. Int. Syst. Comp. 835, 10 (2019)

6. https://iitk.ac.in/ee/ControlOfCyberPhysicalSystems/pics/Cyber-PhysicalSystems_CPS.png

7. http://industry4.sk/technologie/\#.

8. N. Jazdi, IEEE. Int. Conf. Aut. Qual. Test. Rob. 1, 4 (2014) 
9. https://lorbrand.com/wp-content/uploads/2017/03/Impact_Rubber_640x480.png

10. J. Fabianova, P. Kacmary, V. Molnar, P. Michalik, Open Engineering 6, 1 (2016)

11. V. Molnar, K. Pacutova, In: 3rd International Conference on Traffic and Transport Engineering (ICTTE) Univ. Belgrade, Serbia 570-575 (2016) 